\title{
Conversion et décadence dans l'écriture de Claudel
}

\author{
Christophe Ippolito \\ University of the Pacific
}

Mon propos ici sera de montrer comment l'imaginaire décadent et ses représentations fin-de-siècle jouent un rôle pivotal, sinon dans la conversion elle-même, du moins dans l'écriture de la conversion et de l'exégèse chez Claudel $^{1}$. On peut ainsi poser l'hypothèse que, audelà de ses présupposés explicitement symbolistes, l'exégèse claudélienne a parfois un caractère scandaleux qui, s'il peut s'avérer nécessaire au but exégétique (puisqu'après tout le scandale est proprement et étymologiquement cette «pierre » sur laquelle est bâtie l'Église catholique), n'est pas sans rappeler les inversions et outrances de la décadence, et semble parfois contredire l'orthodoxie catholique. Cette ambiguïté était déjà présente dans le discours de la conversion, déchiré entre ses modèles décadents et canoniques, et n'est jamais totalement résolue. Au contraire, Claudel semble jouer sur l'ouverture de l'Écriture à l'écriture, comme on le vérifiera en réfléchissant notamment sur $\mathrm{A} u$ milieu des vitraux de l'Apocalypse et Un poète regarde la croix; son inquiétude catholique semble parfois conduire à refaire l'Écriture sinon la création avec de nouvelles images importées du sociolecte décadent.

Qu'ont en commun nombre d'écrivains de la fin de siècle, sinon la conversion au catholicisme? Claudel (1886) appartient à une lignée qui va de Bloy (1869) à Wilde (1900), et qui comprend entre autres Nouveau, Tolstoï, Charles de Foucauld, Rolland, Huysmans, Coppée, et Joergensen. Suivront après 1900 (Wilde), Bourget (1901), Francis Jammes, Adolphe Retté et Maritain (1905-6), Péguy (1906-10), Massignon (1908), Max Jacob (1909), Jacques Rivière, Alain-Fournier, Ernest Psichari (1913), et Henri Ghéon (1915). L'épisode de la conversion est dans la littérature décadente un lieu du discours extrêmement organisé, à partir des textes canoniques que sont d'une part Les Actes des Apôtres [IX, 1-19], qui raconte l'appel de Damas et la conversion de Saul (où, faut-il le rappeler, la métaphore de la vue perdue et retrouvée organise le texte), d'autre part La Première épître aux Corinthiens [1, 18] qui 


\section{Christophe Ippolito}

introduit l'idée que « le langage de la croix est [...] folie pour ceux qui se perdent, mais pour ceux qui se sauvent, pour nous il est puissance de Dieu ». « Car ce qui est folie de Dieu est plus sage que les hommes », écrit Paul. "Mais ce qu'il y a de fou dans le monde, voilà ce que Dieu a choisi pour confondre les sages. » Enfin s'impose la révision doctrinaire du Père de l'Église le plus rhétoricien qui fût : dans Les Confessions (VIII, 12), le "Prends et lis " s'oppose à « l'amour de l'amour » (III, 1), qui anime l'éveil de la sensualité d'Augustin à Carthage. Augustin rouvrant le livre de l'Apôtre y voit une condamnation de la concupiscence. Là encore, la vue, et en particulier la lecture silencieuse, par opposition à la lectio comme lecture à haute voix, subie, occupe une place centrale. Les deux modèles chrétiens et littéraires sont donc saint Paul et saint Augustin.

Claudel admirait Bloy, dont la conversion-spectacle n'est pourtant pas la plus claire, comme on le lit entre les lignes de sa correspondance avec Villiers et Huysmans. Le chapitre XIII du Désespéré de Léon Bloy, publié en 1886, l'année même de la conversion de Claudel, convertit en hyperboles ce que saint Paul appelait la « folie de Dieu »: « Un double abîme s'ouvrit en cet être, à dater de ce prodigieux instant. Abîme de désir et de fureur que rien ne devait plus combler ». Rhétorique de l'outrance que suit l'évocation toute baudelairienne d'une alchimie créatrice : « À force d'amoureuse foi, il se fit de l'éternité palpitante avec une poignée de temps pétrie dans sa main et se fabriqua de l'espérance avec le plus amer pessimisme [...]. Plus que jamais, il fut un désespéré, mais un de ces désespérés sublimes qui jettent leur cœur dans le ciel, comme un naufragé lancerait toute sa fortune dans l'océan pour ne pas sombrer tout à fait, avant d'avoir au moins entrevu le rivage ${ }^{2}$. Et Bloy, converti par Barbey d'Aurevilly, donnera explicitement saint Augustin pour son modèle dans une lettre à son futur confesseur, le père Millériot, un jésuite. Comme saint Augustin, il retrace un itinéraire tourmenté, et montre que ses errements d'avant la conversion sont dûs d'abord aux exemples blasphématoires de son temps et aux excès naturels de son tempérament. Comme pour l'évêque de Carthage, c'est la lecture (en 1870) du Nouveau Testament qui précipite la conversion. Comme chez les deux saints, la vision joue un rôle essentiel ${ }^{3}$.

Au contraire, Huysmans, dans En Route et la préface de A Rebours (écrite en 1903), décrit la conversion comme une pente insensible, jalonnée de bornes, même si le moment crucial reste soudain, indéterminé, inexplicable. Durtal, dans En Route, avoue : «Il n'y a pas eu de chemin de Damas, pas d'événements qui déterminent une crise ${ }^{4}$ 


\section{Conversion et décadence dans l'écriture de Claudel}

(21). Il relève des débuts d'explication, tels que l'amour de l'art, l'hérédité (sa famille hollandaise très fervente), l'ennui de vivre ${ }^{5}$ (22), et son exaltation à l'idée des monastères ${ }^{6}$. À rebours est en 1903 analysé dans la deuxième partie de la préface " du point de vue de la Grâce "? Entre 1884 et la conversion de la Trappe, il y a « la liturgie, la mystique, l'art " 8 ; les séjours dans les églises, puis une première prière et " l'explosion se fit. Tout cela parait, pour les gens qui ne croient pas à la Grâce, fou ${ }^{9}$. Et Huysmans de revenir lui aussi, comme saint Paul, sur cette « folie de Dieu », comme il revient, à l'instar de Bloy, sur « cette alchimie divine, [...] cette transmutation de la créature humaine en Dieu »10. Adolphe Retté, inspiré par Huysmans dont il a plus tard critiqué les livres catholiques, recompose dans Du Diable à Dieu, Histoire d'une conversion, une version plus orthodoxe du type de conversion qui saisit et illumine. Après avoir lu les premiers chants du Purgatoire dans la Divine comédie de Dante, il " sentit un frisson " puis se mit à trembler, ébloui comme par une lumière intérieure. Pour lui, il y a dans ces vers plus qu'une "magnifique fantasmagorie »11. On voit qu'il combine ici Paul et Augustin.

C'est dans la Revue de la Jeunesse du 10 décembre 1913 (le texte a été repris dans Contacts et Circonstances, 1940) que Claudel, comme on sait, met rétrospectivement en scène sa conversion, qui se nourrit d'abord, au-delà de sa lecture de Joseph de Maistre, d'un terreau purement littéraire. Il me paraît difficile de revenir sur un débat de fond qui a été animé par Charles du Bos ou Henri Guillemin, ou plus récemment par Gérald Antoine. C'est sous l'angle limité de la rhétorique de la décadence que je voudrais analyser ce texte. Comme Claudel l'écrira dans une lettre de 1906 à Gide, " la Vérité catholique s'apprend le mieux, non pas théoriquement par le seul mouvement de la tête qui écoute, mais pratiquement par le placement de toute notre personne dans son ordre vrai, comme un mot qui est mis à sa place, par l'orientation dans le site, par le service dans le corps ${ }^{12}$ (je souligne). Analyser l'épisode reconstitué dans Ma conversion selon ces lignes permet de comprendre la place du discours rhétorique de persuasion, qui reprend plusieurs des morceaux de discours cités plus haut, dans l'écriture autobiographique et sa dispositio. Le préambule consiste en une lumineuse opposition, qui met la fin de siècle en perspective, entre Renan, le naturalisme, le néo-kantisme et les pompes de la troisième république d'un côté et la révélation du Rimbaud des Illuminations de l'autre. "Que l'on se rappelle ces tristes années 80 , l'époque du plein épanouisssement de la littérature naturaliste ${ }^{13}$, écrit 


\section{Christophe Ippolito}

Claudel qui parle dans la même page du « bagne matérialiste »de son lycée, à l'époque où « Renan régnait ».

1886, comme on sait, c'est aussi l'année du manifeste symboliste de Moréas. Une date charnière que 1886 , où dans un livre récent ${ }^{14}$, JeanFrançois Six fait commencer le vingtième siècle. Symbolistes, décadents, et la future garde de la NRF ont en commun le rejet de l'idéologie rationaliste anti-cléricale officielle fondée sur le néo-positivisme des lendemains qui doivent nécessairement chanter, et emmenée par Littré, Taine, Renan, Renouvier ou Burdeau (professeur de philosophie de Claudel). On sait ce que le développement de cette idéologie doit à l'opportunisme politique des fondateurs de la Troisième République en mal de mythes fondateurs, un opportunisme qui prend pour cible les valeurs catholiques de droite qui appartiennent largement au passé, ces mêmes valeurs " inactuelles " qui seront condamnées par Léon XIII. Cette idéologie, comme le remarque René Rémond, « exclut tout ce qui n'est pas rationnel : le sentiment, l'invisible [...]. À plus forte raison le surnaturel qui échappe aux lois de la nature, seule connaissable.

Le symbole est inférieur au concept et le raisonnement par analogie ne tient pas devant le principe d'identité "15. Qui ne voit qu'ainsi l'idéologie dominante exclut tout ce qui n'est pas de près ou de loin rattaché au scientisme? Si le naturalisme zolien est accepté à cause de ses prémisses pseudo-scientifiques, tout ce qui n'est pas dans la norme est rejeté. Le système d'exclusion provoque chez ceux qu'il exclut des rapprochements, des parallélismes, des regroupements, parfois de circonstance, parfois plus durables (NRF, Académie Goncourt...). La vertu de ces compagnonnages de route est de mêler les opposants. C'est aussi le sens des correspondances croisées des années postérieures, telle celle de Massignon et de Claudel. La correspondance de Claudel avec l'auteur du Crépuscule des Dieux porte témoignage de l'atmosphère intellectuelle de l'époque : "Mon but a été de délivrer l'esprit humain de l'horrible et ignoble esclavage dont j'ai tant souffert dans ma jeunesse, celui des prétendues " lois scientifiques ", et de leur déterminisme inflexible, l'affreuse machine des Renan et autres scélérats, de rendre au monde la fraîcheur à jamais virginale de son innocence et de sa nouveauté dont ces misérables l'avaient voulu dépouiller ${ }^{16}$. Enfin, rappelons qu'aux Illuminations et à Une saison en enfer publiés en 1886 succède la publication par Eugène Crépet des Euvres posthumes et des Journaux intimes de Baudelaire en 1887. 


\section{Conversion et décadence dans l'écriture de Claudel}

Les pompes de la religion, le 25 décembre 1886 à Notre-Dame, sont d'abord une occasion de réécriture des auteurs de la décadence : « [...] dans les cérémonies catholiques, considérées avec un dilettantisme supérieur, je trouverais un excitant approprié et la matière de quelques exercices décadents $» 17$. Mais c'est la surprise, le foudroiement sur le chemin de Damas : «En un instant mon cœur fut touché et je crus »18. Puis, sur le modèle augustinien, la révélation est liée à la mémoire (« reconstituer les minutes ", "mémorable jour " ${ }^{19}$ ), mais la longue résistance à la conversion suit le modèle de Huysmans, alors que l'identification finale se fait avec saint Paul : "C'est à moi, Paul, entre tous, que [Jésus] s'adressait et il me promettait son amour " ${ }^{20}$. L'ordre du récit de conversion est ainsi délimité par un jeu dialectique de renvois subtils entre imaginaire décadent et textes canoniques. Ce que Claudel décrit ici, c'est aussi comment la littérature de l'époque a induit chez lui un renversement où les textes sacrés catholiques deviennent le terreau de l'écriture. Il est clair qu'il faut faire la part dans ce texte, comme on sait, du prosélytisme, voire de l'aspect pédagogique que commandait la parution dans une revue destinée à la jeunesse et dirigée par un dominicain, le R.P. Barge. Ce texte extrêmement travaillé, exemplaire à bien des égards, se trouve rappeler, résumer des modèles littéraires moins connus, voire oubliés en 1913. Il résume l'itinéraire d'une génération plus qu'il ne met en exergue l'originalité d'une démarche individuelle. On peut y voir un manifeste intellectuel et littéraire à rebours, qui vient effacer, un quart de siècle après, le manifeste symboliste de Moréas, contemporain de la conversion claudélienne.

Deux autres textes importants traitent de la conversion. Le premier est antérieur de trois ans à celui cité supra. Dans la Troisième Ode, intitulée "Magnificat », et dont le début raconte le moment de la conversion en insistant sur sa soudaineté, il n'y a pas trace de l'imaginaire décadent, sauf à considérer le recours à l'hyperbole et certaine figure féminine rappelant la Vénus païenne de Botticelli : "Toute la Terre Promise lui apparaît dans une lumière éclatante et comme une pucelle neuve, / Toute verte et ruisselante d'eaux comme une femme qui sort du bain "21. Au contraire, il y a profonde séparation, et mise à distance, dans un verset tel que celui-ci : "Ce n'est point mort qui vainc la vie, mais vie qui détruit la mort et elle ne peut tenir contre elle »22. Michel Malicet a réédité et commenté une troisième version de la conversion, extraite des Lettres à l'Ange gardien écrites en 1937. Il remarque justement que dans ce récit « l'essentiel [...] manque, le sentiment de la présence d'un Dieu 


\section{Christophe Ippolito}

personnel, de son innocence, de son «éternelle enfance », son appel à ce nouveau Paul $» 23$. Ce que Malicet appelle l'essentiel, n'est-ce pas ce qui faisait la force du «manifeste » de 1913, et devait emporter la conviction des lecteurs de la revue? De même pour la notion de résistance, gommée en 1937 dans ce texte écrit pour soi : " A voir que tout me donnait tort, je ne pouvais pas avoir le moindre doute d'avoir raison "24 [373]. La deuxième partie de cette phrase est barrée sur le manuscrit. Au-delà de l'orgueil relevé par Malicet, attachons-nous à retrouver dans ce texte les éléments épars d'une écriture de la décadence dans la continuité d'une esthétique de l'outrance, de la démesure, de l'« ubris ». Lorsque Claudel sort de la cathédrale, il «tombe " dans un espace infernal de la Chute qui lui renvoie sa propre image : « les pieds sur cette espèce de miroir luisant, l'asphalte étoilé de flammes rouges que formait l'esplanade sous la pluie ${ }^{25}$. C'est l'image du croyant damné qu'on retrouve dans les propos de Flaminius, dans les Conversations dans le Loir-et-Cher; et cette image est un symbole de la condition du converti en devenir : «Ne peut-on imaginer que le feu signifie un élément permanent de décomposition, un équilibre douloureux obtenu sur une espèce de porte-à-faux, comme un homme qui se tiendrait debout sur un pied foulé, un nouvel équilibre violent et en quelque sorte caricatural, l'homme assis sur le défaut qu'il s'est choisi; en somme, oui, une véritable décomposition (en prenant le mot composition au sens pictural) $\gg 26$. Sa foi nouvelle est comparée à « cette espèce d'énorme enfant entre les bras, cet informe paquet d'absurdes et révoltantes certitudes, [...] ce qu'on venait de me tomber entre les bras de choses folles, révoltantes, désirables, indubitables [...]»27. Plus loin, l'arsenal des adjectifs continue, l'Évangile est une histoire « 'sublime, splendide poignante' et incroyable ». Il y a également quelque chose de satanique dans la description de son monde intérieur, assimilé dans la même page à un «paysage en construction » : «ce monde intérieur en moi, plein d'éruptions, et de mouvements, de marécages, de refus, de violences, de protestations, d'indignations et de sources dans tous les sens ${ }^{28}$. On sait que Claudel était lecteur du Paradis perdu de Milton : deux ans après avoir écrit ce texte, Claudel note dans son journal «le vers fameux où Satan prend conscience de l'enfer qu'il porte en lui ${ }^{29}$.

Passons maintenant à l'exégèse, où parfois transparaissent les germes du doute et de l'inquiétude, voire la tentation de la décadence. On retrouve la même fascination de la chute de l'Ange dans le premier commentaire de l'Apocalypse écrit de 1928 à 1932, et qui prend la forme d'un dialogue entre père et fille. Que ressort-il de ce dialogue? Que c'est 


\section{Conversion et décadence dans l'écriture de Claudel}

avec Lucifer et sa chute que commencerait l'histoire, et que si l'on regarde l'histoire du monde « [par] le dedans, du côté [...] des figures, des symboles efficients $\gg 30$, on peut analyser le temps historique comme répétition de la chute de Lucifer ${ }^{31}$. Et Claudel de prendre plusieurs exemples de cette répétition de la Chute (Adam, le Déluge, mais aussi la Révolution française ou le bolchevisme, " et un tas d'autres événements jusqu'à l'intérieur de la vie de chaque particulier ${ }^{32}$ ). Il arrive à cette conclusion qu' " il y a dans l'Univers non pas conséquence, mais image, de la Chute. Que signifie d'autre le Principe de Carnot et cette dégradation universelle qui est le principe moteur de tout? car la Nature descend : elle transforme au moyen d'une déperdition, mais elle ne peut rien créer par elle-même ni ajouter à sa propre quantité ${ }^{33}$. Négation de l'histoire, pessimisme tragique dans ce drame à deux voix, à propos de "cette littérature apocalyptique embaumée dans ses parfums suspects "34 (137). S'agit-il ici, revenant à saint Augustin (Confessions, X, 6-19), de redire que la création depuis la Genèse est sans cesse traversée d'un besoin de signification? ou s'agit-il de retrouver les « idées primordiales", comme dans le Manifeste de Moréas? C'est bien en poète, et en poète nourri par le symbolisme, que Claudel, exprime, plus qu'il n'explique, un sens. L'image des parfums suspects a une double fonction : elle réfère à Baudelaire, sans doute, mais elle concède aussi le fait que parmi les textes à commenter, Claudel a fait le choix du corpus traditionnellement le plus sujet à caution. Il est vrai que si Claudel a choisi d'écrire sur l'Apocalypse, c'est aussi parce que la Révélation est dans la Bible ce qui s'oppose le plus à l'idéologie positivo-opportuniste des années 1880 , pour laquelle la vérité fondée sur la connaissance du monde repose sur un ensemble de lois démontrables et justifiables. Il est également vrai que le commentaire de l'Apocalypse lui donne une plus grande marge de manœuvre, puisque de son propre aveu, il y voit l'opportunité d'un symbolisme libéré du discours contraint du réel et des faits contraignants dûment enregistrés par les historiens :

[...] ce qu'il y d'intéressant dans l'Apocalypse, comme il s'agit du futur, c'est que là, à la différence des autres livres de la Bible, la réalité n'est pas encore venue fournir d'interprétation temporelle à ces figures dans le ciel écrites. Elles sont là à titre d'images pures et de chiffres efficients, des espèces d'étalons symboliques. Et par là nous ne pouvons douter que dans le reste de la Bible il 


\section{Christophe Ippolito}

ne s'agisse aussi, comme saint Paul nous en avait prévenus, de figures et de symboles, encore que désormais incorporés, remplis par l'histoire et par le fait ${ }^{35}$.

Il reste que le plus troublant est bien l'exacerbation du rôle de l'image de l'idole pourrait-on dire - , qui est ici plus qu'une simple illustration, et qui a une importance en elle-même. D'où l'ironie de la question (rhétorique) suivante : «Pourquoi, au lieu de t'adresser à moi, ne prends-tu pas une de ces belles bibles modernes, à l'imitation des protestants, pleines d'images sérieuses et de documents instructifs ? »36. Pour Claudel, parti de France avant l'évolution du mysticisme des années 1880 vers le satanisme et l'occultisme, et avant les remises en question intellectuelles par exemple exprimées par les Jeunes Turcs de la Revue de métaphysique et de morale, la période de formation intellectuelle est bien celle des débuts du catholicisme esthétisant, même si le Claudel des années 1920 est bien loin de cet esthétisme gratuit qui s'attache aux apparences du décor. Il reste que l'image de la conscience du mal (sinon "dans le mal ») est guide, fil directeur, métaphore filée herméneutique comme il apparaît nettement dans cette récapitulation proposée par le personnage de la fille :

La FILLE : Comme la chute de Satan a été le premier Événement, c'est donc elle qui inaugurerait l'histoire du Monde, la série des événements enfilés sur un trait continu qui se suivent et des catastrophes qui se répondent, comme un fleuve interrompu de temps en temps par des cataractes 37 .

Satan tout au long de ce texte est pris comme une métaphore filée de l'humain, comme si cette métaphore avait un rôle herméneutique dans l'exégèse. Mais l'on est tenté de s'interroger, à l'instar du curé de Chavignolles : «si l'on voit partout des métaphores, que deviendront les faits? " ${ }^{38}$. Certains commentateurs ont voulu distinguer entre celui qui est "d'abord un Poète " et les simples exégètes « orthodoxes ". Jacques Petit cite ainsi le Journal de 1947, où Claudel a retranscrit cette pensée de saint Basile : "Pour moi, l'eau, c'est de l'eau, et quand on me parle de plantes, de poissons, de bêtes, je prends les mots pour ce qu'ils signifient ». "Tant pis pour lui ", commente Claudel. « Les mots signifient, mais les choses elles-mêmes, qu'est-ce qu'elles signifient? "39 Il semble que pour Claudel 


\section{Conversion et décadence dans l'écriture de Claudel}

le travail philologique et doctrinal sur les mots de l'exégète devienne liberté grande de recréation poétique, au-delà des mots, à partir des réseaux symboliques. Claudel bâtit des correspondances, comme Baudelaire le faisait à propos d'une autre œuvre narrative, La Tentation de saint Antoine de Flaubert, qui a eu une profonde influence sur la décadence :
[...] je voudrais surtout attirer l'attention du lecteur sur cette faculté souffrante, souterraine et révoltée, qui tra- verse toute l'œuvre, ce filon ténébreux qui illumine, - ce que les Anglais appellent le subcurrent, - et qui sert de guide à travers ce capharnaüm pandémoniaque de la solitude 40 .

Le « subcurrent » est, selon la définition commune, un courant obscur ou secondaire. Cependant, étant donné que le préfixe « sub-» n'est pas nécessairement équivalent à « de seconde classe », la désignation peut être chez Baudelaire un moyen puissant d'insister sur ce qui est caché mais important - il y a dans ce texte de Baudelaire l'idée d'un sens caché qui nécessiterait une lecture herméneutique, telle que celle que Claudel, ici en accord avec bon nombre d'auteurs du dix-neuvième siècle qui cherchent à inclure des réseaux de micro-éléments particulièrement signifiants derrière le voile de leur discours supposément principal, semble ici appeler de ses voux. Dans son Art poétique, Claudel ajoute que « l'image n'est pas une portion du tout, elle en est le symbole "41. Faudrait-il voir ici l'empreinte de saint Paul, pour qui le sens caché des choses peut être révélé par le symbole, perle au sein de la nacre? Dans tous les cas, le symbole n'est que la partie la plus visible du raisonnement par analogie que Claudel entreprend délibérément. A la pensée linéaire et finaliste de son interrogatrice, le personnage du père répond par une célébration de l'analogie, où les idées ne se suivent pas mais sont « jetées ensemble », donnant proprement naissance aux symboles (étymologiquement, ce qui est jeté ensemble), « comme un flambeau dans une chambre à miroirs »: La FILLE : Pourtant ce mouvement dans le temps aboutit fatalement à Dieu in excelsis, tandis que l'antique dégringolade en précipitait l'Ange pervers.

LE PÈRE : Peut-être il en est la contrepartie, le reflet réel et la copie à rebours. Ascensura est de abysso. Un texte 


\section{Christophe Ippolito}

dit : de mare et un autre texte : de terrâ. [...] Pourquoi veut-on toujours que les idées se suivent? est-ce qu'elles ne peuvent pas se multiplier de tous côtés par voie de reflet, comme un flambeau dans une chambre à miroirs ${ }^{42}$ ?

Dieu « copie à rebours » du diable ? Outre les résonances huysmaniennes se fait jour ici une esthétique de l'inversion et de la réversibilité là encore très proche de Baudelaire : «Depuis la Création il ne s'est jamais agi d'autre chose que de la Fin du Monde ${ }^{43}$, répète le personnage de Claudel.

Si maintenant l'on met $\mathrm{Ma}$ Conversion en rapport avec un passage stratégiquement situé au deuxième paragraphe de Un poète regarde la croix (1938) qui traite de Marie-Madeleine, on peut aisément s'apercevoir du fait que le même jeu dialectique subsiste. Le « parfum précieux » versé par Madeleine est interprété par Claudel comme une figure de l'Évangile, et « comme une effusion énorme tout à coup de délices et de larmes ». La conversion est aussi stratégique, en ce qu'elle ouvre une voie royale pour Claudel comme pour les autres auteurs cités pour résoudre, au moins en apparence, les contradictions, les transferts, les tentations et les outrances de la décadence. On passe vite, comme chez bon nombre d'auteurs fin-de-siècle, de l'Écriture au scandale de la réécriture subversive, comme on peut le voir dans cet extrait de Un poète regarde la croix :

Cette bonne odeur, par exemple, qui remplit la salle du Festin quand Madeleine eut brisé le vase de parfum précieux au-dessus de la tête du Sauveur, n'est-ce pas la même que les prêtres, deux par deux, vont récupérer et respirer, le Jeudi saint, au fond de ces ampoules d'or que la main du Pontife a consacrées? Nardi pistici, nous dit l'Évangile de saint Marc. Une essence qu'il nous faut acheter de confiance et sur la parole du marchand, car, de peur qu'elle s'évapore, elle nous est délivrée dans un vase sans ouverture, la fontaine scellée de nos délices, un paradis hermétique et garanti, tout un jardin concentré dans l'albâtre sprirituel. Et un autre texte exhibe la lecon Nardi spicati, le nard distillé, non pas de la feuille mais de l'épi même, ces grains qui, de la fleur, ont thésaurisé l'arôme le plus exquis. C'est cette ampoule royale qui 
s'écrase et ruisselle entre les doigts de la Madeleine comme une rose irrésistible, comme un cœur qui éclate, au centre de ce comité, autour de l'hôte méconnu, de juges et de professeurs, comme une effusion énorme tout à coup de délices et de larmes. C'est l'Évangile de toutes parts qui s'échappe du récipient immolé et qui remplit toute l'Église et toute la terre, cette sépulture sur Jésus de la mort qui complète celle du baptême. Le Christ, pour son baptême, a eu le plus saint des enfants de la femme, mais pour son sacre, précédant sa suprême intronisation, c'est une pécheresse qu'Il a choisie.

Continuons à suivre attentivement et minutieusement l'Office en notant sur une feuille de papier tout ce qui vient à notre rencontre. 44

Ici encore, c'est de l'entrelacement des figures, en l'occurrence des comparaisons et des métaphores, que naît ce qu'on pourrait appeler la dérive transgressive décadente. C'est alors que la norme tend à devenir parole vide, rhétorique. Le lexique sensuel (fontaine de délices, paradis hermétique et garanti, jardin concentré) pourrait être celui du Jardin des supplices d'un Mirbeau. Sous l'ampoule d'or, on lit un fruit défendu qui ressemble à s'y méprendre à la représentation décadente conventionnelle d'une forme de plaisir charnel, où il est d'ailleurs hasardeux d'assigner une identité masculine ou féminine à la fontaine, au paradis et au jardin (traditionnellement du côté féminin, cependant), ou au ruissellement, au cœur qui éclate entre les doigts de Madeleine. La tentation, ici, est celle d'une inversion de la parole réglée de l'Écriture. Quant à Madeleine, elle est une figure essentielle de la décadence, où elle représente un mythe littéraire. Comme l'écrivit Huysmans sur ce courant littéraire, « [les] poètes lâchèrent Vénus pour la Vierge et ils traitèrent les Bienheureuses comme des Nymphes. Aux déités du Paganisme, si longtemps choyées par le Parnasse, on substituait sainte Madeleine "45. Le choix de MarieMadeleine peut s'expliquer de la manière suivante dans le contexte de la décadence : Marie-Madeleine, sainte prostituée selon l'oxymore baudelairien, est une figure oxymorique et une figure de l'inversion, deux procédés chers aux décadents.

C'est ainsi que la conversion n'efface jamais totalement les tentations décadentes. L'œuvre claudélienne se sert de ces tentations non tant comme repoussoir que comme matériel figuratif, telle une gargouille aux tours d'une cathédrale. S'il est vrai que cette tentation de la déca- 


\section{Christophe Ippolito}

dence n'est pas au centre du message claudélien, elle en est un des soubassements littéraires, souvent renié mais toujours présent. En ce sens, la conversion est aussi pour le jeune Claudel une porte de sortie de la décadence vers un futur littéraire, autant que vers le vingtième siècle ou un autre " positivisme » conservateur néo-chrétien. Littérairement, la conversion abrite l'écriture au sein d'une réécriture de l'Écriture. C'est une position d'autorité qui permet à la fois, pour revenir au sens latin, la traduction et le détournement, voire la révolution complète, de l'élément décadent dans un jardin rhétorique ordonné.

\section{Notes}

1 Une première version de cet essai a été présentée sous forme de communication à la réunion de la «Paul Claudel Society » lors de la conférence annuelle de la « Modern Language Association » à Chicago le 29 décembre 1999.

2 Le Désespéré (Paris : Mercure de France, 1933), 44-46.

3 Cf. l'épisode où Marchenoir surprend Véronique en pénitente : "L'effet de cette vision fut de transformer immédiatement la fureur de Marchenoir en une compassion déchirante » (Le Désespéré [Paris : Mercure de France, 1933], 309).

4 En Route (Paris : Plon, 1961), 21.

5 Id., 22.

6 Id., 69.

7 A Rebours (Paris, Folio/Gallimard, 1977), Préface écrite vingt ans après le roman, 73.

8 Id., 74.

9 Id., 75.

10 En Route (Paris : Plon, 1961), 46.

11 Adolphe Retté, Du Diable à Dieu, Histoire d'une conversion (Paris : Albert Messein, 1901), 59.

12 Paul Claudel et André Gide, Correspondance 1899-1926, Préface et notes par Robert Mallet (Paris : Gallimard, 1949), 66.

13 Paul Claudel, Euvres en prose, Bibliothèque de la Pléiade, Préface par Gaëtan Picon, Textes établis et annotés par Jacques Petit et Charles Galpérine (Paris : Gallimard, 1965), 1009. 


\section{Conversion et décadence dans l'écriture de Claudel}

14 1886, Naissance du vingtième siècle (Paris : Seuil, 1886).

15 « 1886 : L'idéologie dominante », in Paul Claudel et la Conversion, Les Cahiers du Rocher 1 (Monaco : Le Rocher, 1986), 64.

16 Jacques Petit, "Paul Claudel et Elémir Bourges. Lettres inédites (1903. 1910) ", Cahiers Paul Claudel 1 (Paris : Gallimard, 1959), 174.

17 Paul Claudel, CEuvres en prose, Bibliothèque de la Pléiade, Préface par Gaëtan Picon, Textes établis et annotés par Jacques Petit et Charles Galpérine (Paris : Gallimard, 1965), 1009-1010.

18 Id., 1010.

19 Id., respectivement 1010 et 1011 .

20 Id., 1011-1012.

21 Paul Claudel, Cinq grandes odes, in Cuure poétique, Bibliothèque de la Pléiade, Introduction par Stanislas Fumet, (Paris : Gallimard, 1957), 260.

22 Id., 253.

23 "Un récit inédit de la conversion ", in Pierre Brunel, éd., Paul Claudel, Les Cahiers de l'Herme (Paris : Éditions de l'Herne, 1997), 374.

24 Id., 373.

25 Id., 372.

26 Paul Claudel, Euvres en prose, Bibliothèque de la Pléiade, Préface par Gaëtan Picon, Textes établis et annotés par Jacques Petit et Charles Galpérine (Paris : Gallimard, 1965), 702. Cité par Pierre Brunel in Claudel et le Satanisme anglo-saxon (Ottawa : Éditions de l'Université d'Ottawa, 1975), 212.

27 «Un récit inédit de la conversion », in Pierre Brunel, éd., Paul Claudel, Les Cahiers de l'Herne (Paris : Éditions de l'Herne, 1997), 373.

28 Id. 373.

29 Pierre Brunel, Claudel et le Satanisme anglo-saxon (Ottawa : Éditions de l'Université d'Ottawa, 1975), 212.

30 Paul Claudel, Au milieu des vitraux de l'Apocalypse, in Commentaires et Exégèses, in CEurres complètes, tome XXVI (Paris : Gallimard, 1967), 18.

31 "[...] c'est la chute de Satan qui a déclenché toute l'histoire du monde " (Id., 135).

32 Id., 19.

33 Id., 21.

34 Id., 137.

35 Id., 13. 


\section{Christophe Ippolito}

36 Id., 36.

37 Id., 21.

38 Gustave Flaubert, Bouvard et Pécuchet, in Curres complètes, collection «L'Intégrale », Bernard Masson, éd. (Paris : Seuil, 1964), tome II, 283.

39 Jacques Petit, «Claudel, lecteur de la Bible », in La Pensée religieuse de Claudel (Paris : Desclée de Brouwer, 1969), 47.

40 Charles Baudelaire, "Madame Bovary ", in Henri Lemaître, éd., Curiosités esthétiques/L'Art romantique et autres cuvres critiques de Baudelaire (Paris : Garnier, 1962), 651.

41 Paul Claudel, Euvre poétique, Bibliothèque de la Pléiade, Introduction par Stanislas Fumet, (Paris : Gallimard, 1957), 186.

42 Paul Claudel, Au milieu des vitraux de l'Apocalypse, in Commentaires et Exégèses, in Euures complètes, tome XXVI (Paris : Gallimard, 1967), 22.

43 Id., 13.

44 Paul Claudel, Un poète regarde la croix, in Commentaires et Exégèses, in CEuvres complètes, tome XIX (Paris : Gallimard, 1962), 182.

45 Cité in Jean Pierrot, L'Imaginaire décadent (Paris : Presses Universitaires de France, 1977), 111. 